\title{
ESTUDO DE LIBERAÇÃO IN VITRO DA CRISINA A PARTIR DE SISTEMA MICROEMULSIONADO PARA USO NA HIPERALGESIA
}

\author{
I.M.M. RAMALHO ${ }^{1}$; F.I.C. DUARTE ${ }^{1}$; S.B. CARNEIRO ${ }^{2}$; L.J. QUINTANS-JUNIOR ${ }^{3}$; \\ B.P.G.L. DAMASCENO ${ }^{3}$; E.A. OSTROSKY ${ }^{1}$, A.A.N. LIMA ${ }^{1}$
}

${ }^{1}$ Universidade Federal do Rio Grande do Norte, Programa de Pós-graduação em Ciências Farmacêuticas.

${ }^{2}$ Universidade Federal do Amazonas, Programa de Pós-graduação em Inovação Farmacêutica.

3 Universidade Federal de Sergipe, Programa de Pós-graduação em Ciências Farmacêuticas.

4 Universidade Estadual da Paraíba, Programa de Pós-graduação em Ciências Farmacêuticas.

E-mail para contato: adleyantonini@yahoo.com.br

RESUMO - Estudos de liberação in vitro de sistemas de liberação de fármacos coloidais são um dos mais importantes métodos de caracterização, pois além de predizer a performance in vivo, o seu perfil pode estar relacionado com a estrutura do sistema. Neste contexto, o objetivo deste trabalho foi determinar o perfil de liberação da crisina a partir da microemulsão (ME) para análise do melhor modelo para esse fenômeno. O estudo de liberação in vitro da ME foi realizado com células de difusão de Franz $(m=3)$. Após 12 horas, foi determinado o perfil cumulativo de liberação usando espectrofotômetro UV visivel. A quantidade de crisina liberada da ME foi 31,21\%. A análise de regressão linear foi feita para determinar o melhor modelo cinético que reflete a liberação da crisina. Três diferentes modelos matemáticos (ordem zero, Higuchi e primeira ordem) foram testados. Os coeficientes de correlação foram 0,9998, 0,9748 e 0,9617. A liberação da crisina em microemulsão seguiu o modelo de ordem zero, representando um perfil de liberação ideal para atingir uma ação farmacológica prolongada. O fluxo no estado estacionário foi $11.218 \mu \mathrm{g} / \mathrm{cm}^{2} \mathrm{~h}$. O lag time para a crisina atingir um fluxo constante de liberação foi $9.78 \mathrm{~min}$. O coeficiente de liberação (Kr) foi calculado utilizando o fluxo e a concentração inicial da crisina, atingindo o valor de $11.218 \mathrm{mg} / \mathrm{cm}^{2} \mathrm{~h}$. Assim, os parâmetros de liberação obtidos para a ME mostraram-se promissores para a realização de estudos complementares e aplicação da ME para o tratamento da hiperalgesia. 
ABSTRACT - In vitro release studies of colloidal drug delivery systems are one of the most important characterization methods since, in addition to predicting in vivo performance, its profile may be related to the structure of the system. In this context, the objective of this work was to determine the release profile of the chrysin from the microemulsion (ME) to analyze the best model for this phenomenon. The in vitro release study of ME was performed with Franz diffusion cells $(m=3)$. After 12 hours, the cumulative release profile was determined using a visible UV spectrophotometer. The amount of chrysin released from $M E$ was $31.21 \%$. Linear regression analysis was performed to determine the best kinetic model that reflects the release of the chrysin. Three different mathematical models (order zero, Higuchi and first order) were tested. The correlation coefficients were 0.9999, 0.9748 and 0.9617. The release of chrysin in microemulsion followed the zero order model, representing an ideal release profile to achieve a prolonged pharmacological action. The steady-state flow was $11,218 \mu \mathrm{g} / \mathrm{cm}^{2} \mathrm{~h}$. Lag time for chrysin achieve a constant rate of release from the ME was $9.78 \mathrm{~min}$. The release coefficient (Kr) was calculated using the flux and the initial concentration of the chrysin, reaching $11,218 \mathrm{mg} / \mathrm{cm}^{2} \mathrm{~h}$. Thus, the release parameters obtained for ME were shown to be promising for the accomplishment of complementary studies and application of $M E$ for the treatment of hyperalgesia.

Keywords: kinetical release, microemulsion, flavonoid.

\section{INTRODUÇÃO}

A Crisina (5,7-Dihidroxiflavona) pertence à classe flavona de flavonoides. É encontrada naturalmente em mel, própolis, e várias espécies de plantas, incluindo espécies do gênero Pelargonium, Passiflora e da família Pinaceae (PICHICHERO et al., 2011).

Inúmeras atividades farmacológicas vêm sendo atribuídas à crisina dentre as quais pode-se citar o potencial anti-inflamatório (BAE et al., 2011), antinociceptivo (FARKHONDEH et al., 2015; ZHAI et al., 2008; RAUF et al., 2015) anticonvulsivantes (MEDINA et al., 1990), antioxidante (PUSHPAVALLI et al., 2010), anti-hipertensivo (VILLAR et al., 2002), antineoplásico (PICHICHERO et al., 2011) e anti-hiperlipidêmico (ZARZECKI et al., 2014). Também há relatos que sugerem que a crisina aumenta os níveis de testosterona pela inibição da enzima aromatase (KAO et al., 1998), que converte a testosterona em estradiol, e em decorrência disso, a crisina já está disponível no mercado como um suplemento dietético (500 mg por cápsula) (iHerbInc., Monrovia, Califórnia; VitaDigest, Walnut, Califórnia).

Apesar das diversas atividades biológicas constatadas, o uso terapêutico da crisina ainda depende da melhoria de seu perfil farmacocinético após administração oral, pois apresenta uma solubilidade aquosa limitada e baixa biodisponibilidade (WALLE et al., 2001). Desta maneira, há a necessidade de desenvolver um sistema de liberação que veicule a molécula terapeuticamente ativa para o local de ação.

Assim, o desenvolvimento de novos sistemas de liberação (NSL) visando a veiculação de moléculas com potencial farmacológico existente é um processo que continua crescendo na pesquisa farmacêutica devido as diversas vantagens destes sistemas como a liberação controlada de fármacos (BILIA et al., 2014). 
O perfil de liberação in vitro de sistemas coloidais contendo fármacos é uma caracterização físico-química muito importante, pois além de prever a performance in vivo, pode ser correlacionada à microestrutura do veículo, descrevendo o comportamento estrutural da formulação em escala microscópica e as possíveis interações entre o fármaco e o veículo (MORAIS; BURGESS, 2014). Neste contexto, o objetivo deste trabalho foi determinar o perfil de liberação da crisina a partir da microemulsão para análise do melhor modelo para esse fenômeno.

\section{MATERIAIS E MÉTODOS}

\subsection{MATERIAIS}

Crisina (Sigma-Aldrich, EUA); Microemulsão contendo: Miristato de isopropila (Farmos, Brasil), PEG-8 cáprico/caprílico glicerídeo (LAS ${ }^{\circledR}$-Brasquim, Brasil), Água deionizada obtida através de osmose reversa (Permution, Brasil); Células de Franz; Membrana de acetato de celulose (Merk, EUA); Etanol PA (Isofar, Brasil); Espectrofotométrico (UV Evolution 60 S, Thermo Scientific, EUA); Banho termostatizado.

\subsection{MÉTODOS}

As cinéticas de difusão in vitro foram conduzidas utilizando-se células de difusão tipo Franz. O compartimento receptor foi preenchido solução etanol/água (1:1) afim de manter as condições sink, num sistema composto de três células individuais, que estavam imersas em um banho termostatizado à $37 \pm 0,5^{\circ} \mathrm{C}$. $\mathrm{O}$ compartimento doador foi preenchido com um volume de $300 \mu \mathrm{L}$ da microemulsão contendo crisina (MECS) $(0,1 \%)$ a ser testada. Para tanto, foram coletadas e repostas $1 \mathrm{ml}$ da solução receptora nos intervalos de tempo determinados e a quantificação de crisina liberada neste compartimento foi feita através de leitura espectrofotométrica no comprimento de 269 nm.

Para a avaliação da cinética, os resultados da liberação in vitro foram compilados em gráficos de dispersão xy, característicos de três modelos cinéticos. A partir dos gráficos, realizou-se análise de regressão linear, e o modelo que apresentou o maior valor de $\mathrm{r}$ foi o selecionado. $\mathrm{O}$ valor de fluxo $(\mathrm{J})$, velocidade de liberação, da crisina foi calculado na porção linear dos pontos experimentais selecionados (PREMARATHNE; KARUNARATNE; PERERA, 2016). O lag time ( $\left.\mathrm{t}_{\text {lag }}\right)$ foi calculado através da extrapolação da reta de regressão linear até o eixo do tempo (CAVALCANTI et al., 2016). O coeficiente de liberação, $\mathrm{Kr}$, foi calculado através da Equação 1 (PREMARATHNE; KARUNARATNE; PERERA, 2016):

$$
K r=\frac{F s s}{C o}
$$

Onde, $\mathrm{Kr}$ é o coeficiente de liberação, Jss é o fluxo no estado estacionário através das membranas de acetato de celulose $\left(\mu \mathrm{g} / \mathrm{cm}^{2} \mathrm{~h}\right)$ e Co é a concentração inicial de crisina na formulação $(\mu \mathrm{g} / \mathrm{mg})$.

Além disso, realizou-se o cálculo do percentual de liberação da crisina tanto da ME ao longo do tempo através da Equação (2) (CAVALCANTI et al., 2016):

$$
\text { Percentual de liberação }(\%)=\frac{R t}{L} \times 100
$$

Onde Rt corresponde a quantidade de crisina liberada no tempo $t$ e $L$ representa a quantidade inicial de crisina na ME. 


\section{RESULTADOS E DISCUSSÃO}

A ME proposta liberou a crisina lentamente ao longo do tempo, tendo em vista que o fármaco se encontra confinado nas gotículas revestidas pelos tensoativos. O perfil de liberação da crisina pode ser observado na Figura 1, que representa a quantidade liberada $\left(\mu \mathrm{g} / \mathrm{cm}^{2}\right)$ em função do tempo para a MECS ao longo de 12 horas de ensaio. Graficamente observou-se que a liberação da crisina do veículo microemulsionado foi contínua-crescente até $12 \mathrm{~h}$, não havendo a formação de platô.

Figura 1 - Perfil de liberação da crisina $\left(\mu \mathrm{g} / \mathrm{cm}^{2}\right)$ em função do tempo (h) a partir da ME.

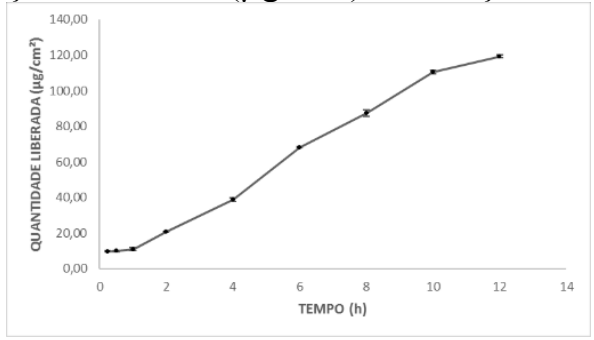

Fonte: Próprio autor

Neste estudo os principais modelos existentes foram aplicados para os resultados da liberação in vitro, sendo estes de ordem zero, pseudo primeira ordem (Higuchi), e primeira ordem. A Figura 2 mostra o gráfico resultante da aplicação desses modelos cinéticos aos dados obtidos pela liberação in vitro da crisina incorporada ao sistema microemulsionado.

Figura 2 - Perfil dos modelos cinéticos testados para a ME.

$$
\begin{gathered}
y=10,147 x+3,5399 \\
r=0,9998
\end{gathered}
$$

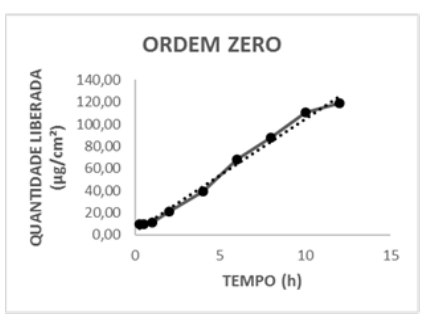

Fonte: Próprio autor.

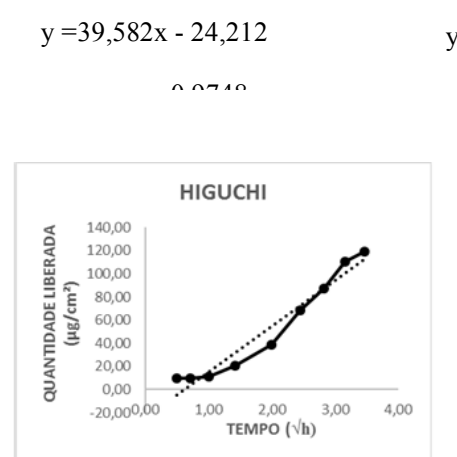

$y=0,1013 x+1,0436$

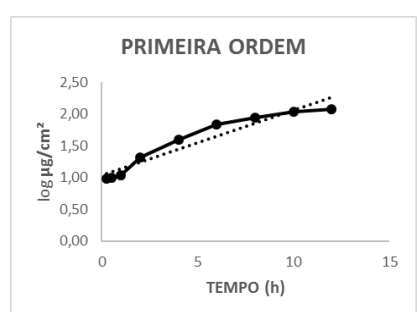

Legenda: Os modelos cinéticos testados foram de Ordem Zero ( $\mu \mathrm{g} / \mathrm{cm}^{2} \mathrm{x}$ tempo), Higuchi $\left(\mu \mathrm{g} / \mathrm{cm}^{2} \mathrm{x}\right.$ raiz do tempo) e Primeira Ordem ( $\log \mu \mathrm{g} / \mathrm{cm}^{2} \mathrm{x}$ tempo).

A liberação da crisina da ME seguiu modelo cinético de ordem zero, como visto pelo maior valor de r. O modelo de ordem zero caracteriza um sistema de liberação que não se desagrega, portanto, o fármaco é liberado lentamente independente da concentração do fármaco (SHAIKH; KSHIRSAGAR; PATIL, 2015). Os resultados dos parâmetros cinéticos obtidos pela ME mostram que o valor de Jss de $11,218 \mu \mathrm{g} / \mathrm{cm}^{2} \mathrm{~h}$ obtido pela crisina contido no veículo microemulsionado foi considerado satisfatório para estudos in vitro. O lag time de 8 minutos para a ME foi considerado baixo, levando pouco tempo para alcançar uma velocidade constante de liberação e, o $\mathrm{Kr}$ demonstrou que em cada tempo (h), 11,218 mg de crisina foi capaz de ser liberada em $1 \mathrm{~cm}^{2}$ da célula, sendo considerado também um resultado satisfatório. Portanto, os constituintes 
da $\mathrm{ME}$, bem como a microestrutura formulada não foram desestruturadas e permitiram uma liberação lenta da crisina através da matriz da $\mathrm{ME}$, fazendo com que houvesse ao longo do tempo o desenvolvimento de um perfil de liberação modificada ideal para atingir a liberação prolongada de fármacos, sendo importante principalmente para algumas classes de medicamentos como analgésicos (SHAIKH; KSHIRSAGAR; PATIL, 2015).

\section{CONCLUSÃO}

O ensaio de liberação in vitro é apenas um indicativo de desempenho da ME, pois ele foi considerado satisfatório, indispensável e esclarecedor para traçar o perfil de influência que os componentes da $\mathrm{ME}$, bem como a maneira que esta foi estruturada, são capazes de influenciar na liberação da crisina em membranas artificiais, sugere-se uma liberação prolongada, mas ainda é necessário mais testes de liberação, com maior disponibilidade de tempo para a afirmação do tipo de liberação, incluindo também mais caracterizações para melhor descrição de sua estruturação. Os resultados dão subsídios para dar continuidade a estudos que visem otimizar e valorizar a formulação para que esta possa ser veiculada, oferecendo mais uma alternativa para o tratamento da hiperalgesia.

\section{REFERÊNCIAS}

BAE, Y.; LEE, S.; KIM, S. H. Chrysin suppresses mast cell-mediated allergic inflammation: Involvement of calcium, caspase-1 and nuclear factor-kappaB.

Toxicology Applied Pharmacology, v. 254, p. 56-64, 2011.

BILIA, A. R. et al. Flavonoids Loaded in Nanocarriers: An Opportunity to Increase Oral Bioavailability and Bioefficacy. Food and Nutrition Sciences, v. 5, p. 12121227, 2014.

CAVALCANTI, A. L. M. et al. Microemulsion for topical application of pentoxifylline: In vitro release and in vivo evaluation. International Journal of Pharmaceutics, v. 506, n. 1-2, p. 351-360, 2016.

FARKHONDEH, T. et al. Effect of chrysin on nociception in formalin test and serum levels of noradrenalin and corticosterone in rats. International journal of clinical and experimental medicineis, v. 8, n. 2, p. 2465-2470, 2015.

KAO, Y. C.; ZHOU, C.; SHERMAN, M.; LAUGHTON, C. A.; CHEN, S. Molecular basis of the inhibition of human aromatase (estrogen synthetase) by flavone and isoflavone phytoestrogens: a site-directed mutagenesis study. Environmental Health Perspectives, v. 106, p. 85-92, 1998.

MEDINA, J. H.; PALADINI, A. C.; WOLFMAN, C.; STEIN, M. L. DE; CALVO, D.; DIAZ, L. E. et al. Chrysin (5,7-Di-OH-Flavone), a naturally-occurring ligant for benzodiazepine receptors, with anticonvulsivant properties. Biochemecal pharmacology, v. 40, p. 2227-31, 1990.

MORAIS, J. M.; BURGESS, D. J. In vitro release testing methods for vitamin e nanoemulsions. International Journal of Pharmaceutics, v. 475, n. 1, p. 393-400, 2014. 
PICHICHERO, E. et al. Chrysin-induced apoptosis is mediated through p38 and Bax activation in B16-F1 and A375 melanoma cells. International Journal of Oncology, v. 38, n. 2, p. 473-483, 2011.

PUSHPAVALLI, G.; KALAIARASI, P.; VEERAMANI, C.; PUGALENDI, K. V. Effect of chrysin on hepatoprotective and antioxidant status in D-galactosamineinduced hepatitis in rats. European Journal Pharmacolpgy, v. 631, p. 36-41, 2010.

PREMARATHNE, E. P. N.; KARUNARATNE, D. N.; PERERA, A. D. L. C. Controlled release of diclofenac sodium in glycolipid incorporated micro emulsions. International Journal of Pharmaceutics, v. 511, n. 2, p. 890-898, 2016.

RAUF, A. et al. Suppression of inflammatory response by chrysin, a flavone isolated from Potentilla evestita Th. Wolf. in silico predictive study on its mechanistic effect. Fitoterapia, v. 103, p. 129-135, 2015b.

SHAIKH, H. K.; KSHIRSAGAR, R. V.; PATIL, S. G. Mathematical models for drug release characterization: A review. World Journal of Pharmacy and

Pharmaceutical Sciences, v. 4, n. 4, p. 324-338, 2015.

VILLAR, I. C.; JIMENOZ, R.; GALISTEO, M.; GARCIO-SAURA, M. F.; ZARZUELO, A.; LUARTE, J. Effect of chronic chysin treatment in spontaneously hypertensive rats. Planta Medica, v. 68, p. 845-7, 2002.

WALLE, T.; OTAKE, Y.; BRUBAKER, J. A.; WALLE, U. K.; HALUSHKA, P. V. Disposition and metabolism of the flavonoid chrysin in normal volunteers. Britsh Journal Clinical Pharmacology, 51:143-6, 2001.

ZARZECKI, M. S. et al. Hypolipidemic action of chrysin on Triton WR-1339-induced hyperlipidemia in female C57BL/6 mice. Toxicology Reports, v. 1, p. 200-208, 2014.

ZHAI, K.; HU, L.; CHEN, J.; FU, C.; CHEN, Q. Chrysin Induces Hyperalgesia via the GABA A Receptor in Mice. Planta Medica, v. 74, p. 1229-1234, 2008.

\section{AGRADECIMENTOS}

Agradecemos a CAPES, UFRN, UEPB e UFS. 